\title{
Airline OR Innovations Soar During COVID-19 Recovery
}

\author{
Laurie A. Garrow ${ }^{1}$ (D) $\cdot$ Virginie Lurkin ${ }^{2} \cdot$ Lavanya Marla $^{3}$
}

Received: 19 September 2021 / Accepted: 5 February 2022 / Published online: 22 February 2022

(c) The Author(s), under exclusive licence to Springer Nature Switzerland AG 2022

\begin{abstract}
The Airline Group of the International Federation of Operational Research Societies (AGIFORS) held four conferences during May to July 2021 that focused on how COVID-19 was impacting and reshaping the airline industry. Dozens of airline representatives from around the world spoke about how fundamental changes in passenger demand and booking patterns are reshaping the airline industry and driving innovation and research needs. Customers are booking much closer to departure and are canceling or exchanging their tickets more frequently than before COVID-19. Volatility in demand has increased as travel restrictions change and borders reopen. Consequently, greater uncertainty in demand forecasts used as inputs to optimization algorithms is motivating the need for new approaches. Revenue management and scheduling departments are innovating how they predict market sizes and exploring ways to use new data sources or historic bookings in forecasting models. Scheduling and operations departments are making many more flight-cancellation and equipment-swap decisions one to three days from flight departure, which is changing the role of recovery planning. New urgency exists within crew to design duties and rosters that are robust to ever-evolving schedules. Across functional areas, the increasing emphasis is to develop integrated solutions that jointly optimize schedules, crew pairings, and crew rosters for demand forecasts that are uncertain at the time rosters are published. This paper describes how these changes are reshaping the airline industry during COVID-19, explains why short lead times for bookings and uncertainty in demand volumes are expected to remain after COVID-19, and describes how the airline industry is innovating and developing new operations research approaches for handling uncertain and volatile demand.
\end{abstract}

Keywords Airlines · COVID-19 $\cdot$ Demand forecasting $\cdot$ Schedule planning $\cdot$ Airline operations $\cdot$ Crew planning

This article is part of Topical Collection on Airline Innovations During COVID-19.

Laurie A. Garrow

Laurie.garrow@ce.gatech.edu

Extended author information available on the last page of the article 


\section{Introduction}

The year 2020 was unprecedented within aviation. Never before has the airline industry - at a global level-experienced such a sharp and sustained decline in air passenger demand. While prior events such as SARS ${ }^{1}$ and $\mathrm{MERS}^{2}$ provided airlines with some experience in dealing with a health crisis caused by a coronavirus, they were in isolated regions of the world and "didn't prepare [us] for dealing with something like this global pandemic" [1]. In the spring of 2020, global demand dropped off by 90 to 95 percent and airlines throughout the world were scrambling to control costs and realign their schedules and business models to help minimize daily cash burn [2]. As of the summer of 2021, at least 40 airlines have ceased or suspended operations due to the COVID-19 pandemic [3].

In 2021, many airlines have moved from crisis mode to recovery mode. Several factors are contributing to the recovery of airline passenger demand-most notably the rollout of COVID-19 vaccines and the reopening of international borders. For example, in July 2021, Ed Bastian, Delta's chief executive officer, noted that in the USA, "domestic leisure travel is fully recovered to 2019 levels and there are encouraging signs of improvement in business and international travel" [4]. Almost all major US airlines, including Alaska, Allegiant, American, Delta, JetBlue, Southwest, and Spirit, reported second-quarter profits in 2021 [4-8].

Move the clock forward by just two months to September 2021, however, and the timeline for US recovery has lengthened as concerns about new variants have emerged. Outlooks for recovery are changing frequently. In its update as of September 4, 2021, Airlines for America reported that since mid-July the growth of overall and corporate ticket sales has declined, in large part due to a resurgence of COVID-19 cases and hospitalizations [9]. As of early September 2021, domestic US air travel was down 17 percent and international air travel was down 43 percent [9].

Outside of the USA, many airlines are experiencing slower recoveries-particularly those that predominately serve international markets. For example, Bertalan Juhasz, senior manager of Operations Research and Insights at Finnair, notes that "our business model relies heavily on ferrying passengers between Europe and Asia, and that just doesn't happen right now" [10]. Mirco Bharpalani, head of Data and Analytics at the Lufthansa Group, notes that "in Europe, we are a bit jealous of our colleagues on the other side of the Atlantic Ocean [given] we don't have a big domestic market and are still [seeing depressed demand] due to all of the travel restrictions that are still in place" [11]. Similar observations on slower recoveries due to the lack of international travel, border restrictions, and unwillingness of passengers and crew to travel to COVID-19 hotspots have been expressed by Emre Pekesen of Pegasus, a low-cost airline headquartered in Istanbul, and Rodrigo Correia of the LATAM Airlines Group, an airline holding company headquartered in Santiago, Chile, with subsidiaries in several Latin American countries [12, 13].

\footnotetext{
1 Severe acute respiratory syndrome-related coronavirus, or SARS-CoV

${ }^{2}$ Middle East respiratory syndrome-related coronavirus, or MERS-CoV
} 
From May to July 2021, the Airline Group of the International Federation of Operational Research Societies (AGIFORS) held four online conferences that focused on how COVID-19 was reshaping the airline industry and driving the need for new research in OR. This paper provides a summary of the technical presentations and panel discussions from these conferences. Some of the conferences, such as the one on revenue management and pricing, focused more on practical perspectives, whereas other conferences, such as the one on crew management, included more academic perspectives.

The balance of this paper is focused on summarizing discussions from these conferences. Innovations from each of the individual conferences is first presented. For the revenue management study group, this includes an overview of fundamental changes in passenger demand and booking patterns and innovations in incorporating new data into demand forecasts. In the schedule planning area, increased focus is being placed on how to schedule flights given high levels of uncertainty in demand. Within operations, the primary role of recovery planning has shifted from canceling or holding flights or swapping aircraft on the day of operation due to weather or mechanical reasons, to continuously canceling, adding, and up-gauging or downgauging flights in order to better match supply with passenger bookings that have actually materialized. Within crew, much attention is being placed on manpower planning and designing duties and rosters that are robust to ever-evolving schedules. Across all conferences, there was a recognition of the need to develop integrated solutions, particularly with respect to jointly optimizing schedules, crew pairings, and crew rosters under demand uncertainty. These and other innovations are presented in the following sections.

\section{Changes in Passenger Demand and Booking Patterns}

Several changes to airline passenger demand and booking patterns have occurred during the pandemic. Some of these changes have been quite dramatic, such as an increase in the percentage of leisure travelers flying; other changes have been subtler, such as the presence of a larger number of inelastic leisure travelers. This section presents an overview of how airline passenger demand and booking patterns have changed since 2019 and industry experts' opinions as to how airline customer segments may further evolve in 2022 and beyond.

Airline passengers traveling during the COVID-19 recovery represent a different mix of customers who have distinct travel needs, particularly with respect to when they want to book their flights. As Juhasz at Finnair notes, "One thing that we have observed is that passengers now book much closer to departure, and I think that will probably stay like that for quite some time, probably many years, as long as there's uncertainty on when [and where] the next wave of COVID infections will come" [10]. Customers' desire to book closer to departure is motivated in part by travel restrictions imposed by different countries that frequently change; these restrictions 
include quarantines, one or more $\mathrm{PCR}^{3}$ tests before and after entry into the country, proof of immunization, and banning entry of passengers on nonessential travel from hotspot areas. As Bharpalani of the Lufthansa Group explains, "Because there is so much uncertainty on what's allowed and what's not allowed, the booking times are really short for this year" [11].

Given the evolving travel restrictions and emergence of new COVID-19 hotspots, airline passengers who are booking and traveling during the COVID-19 recovery need the ability to easily make changes to their flights and travel plans. Many airlines have waived ticket exchange and cancellation fees, and many within the industry are expecting that these flexible exchange and cancellation policies will remain after the pandemic. As Pekesen notes, Pegasus Airlines is currently allowing passengers to make an unlimited number of free exchanges on their tickets and expects that "after the pandemic, consumers would like to continue to have this freedom...which presents a new challenge for us" [13]. Correia of LATAM Airlines Group observes that "due to these unprecedented times, we're seeing the industry offering a lot of flexibility, and it's hard to tell if the desire for flexibility will remain going forward" [12].

During COVID-19, airlines have experienced high degrees of uncertainty in where and when demand will materialize. According to Terrance Bradshaw, American Airlines is seeing volatility in its international demand due to changes in various travel restrictions and the ability to fly into different countries. In this environment, he explains, "We're trying to be as reactive as we can, as we observe the day-to-day, sometimes hour-to-hour changes that happen" [14]. Juhasz at Finnair also says that "demand is changing so quickly, especially on certain routes, that you really have to monitor [the daily booking reports that contain information on] daily bookings, daily cancellations, and rebookings" [10]. The inability to predict demand with a high degree of certainty makes it challenging for airlines to align their schedules and resources to serve demand ${ }^{4}$.

A sense also exists that after COVID-19 there will be a new normal, further complicating the ability to create accurate forecasts using historic data, as the historic data are no longer representative of current demand. For example, the mix of air travelers during the COVID-19 recovery differs from that before the pandemic, with leisure travel recovering faster than business travel-particularly in domestic markets that have few travel restrictions. Some airlines, especially low-cost carriers, have actually continued their expansion plans and/or have entered new markets. In 2021 JetBlue is adding 24 new routes across the USA and Central America and initiating service in Miami, Key West, Guatemala City, and Los Cabos [16]. Pegasus Airlines, a low-cost carrier based in Istanbul, also started new flights in 2021 to leisure destinations, including Antalya and Dalaman [13]. Within the USA, three airlines are deploying more capacity in October 2021 than in 2019; these include

\footnotetext{
3 Polymerase chain reaction, or the molecular-level detection of COVID-19's genetic material.

4 For additional information on how the impact of travel restrictions on airline operations and passenger demand is driving the need for unprecedented flexibility and speed in decision-making processes within airlines, see [15].
} 
Allegiant (up 25.3 percent), Spirit (up 14.2 percent), and Frontier (up 1.0 percent) (reported by [9] using published schedules from Diio by Cirium).

More subtle changes to the passenger mix traveling during recovery have also occurred. As Alex Matson of Alaska Airlines explains, "The leisure passenger that we're seeing now is definitely not a typical leisure passenger...people have a lot of travel credits, travel awards, and a huge amount of paid time off they have saved up, which means that we're seeing a lot more inelastic leisure passengers who are traveling in ways that they wouldn't have prior to the pandemic" [17]. Mixed trip purposes are also becoming more common, e.g., "People may go to a nice island in Spain and work there for two weeks, combining travel and work" [11]. Developing OR solutions to optimize for these and other new demand segments post-COVID-19 will be important [14]. For example, after the pandemic, telework and teleconferencing may create new demand segments. As Bharpalani of the Lufthansa Group suggests, telework may enable individuals to live further from their offices and fly to the office once a month or so [11].

In summary, COVID-19 has led to fundamental changes in airline passenger demand, and many of these changes are expected to remain after the pandemic. Some of these changes, most notably when passengers purchase their tickets and how often they change their tickets, are particularly challenging for airlines as they impact multiple functional areas. Given the importance of understanding how airline passenger demand is changing during and after COVID-19, many innovations are occurring in the industry with respect to predicting demand. These innovations and research needs are discussed in the next section.

\section{Infusing New Data Into Demand Forecasts}

A well-calibrated demand forecast model is at the core of any revenue management system and schedule planning models. Traditional demand forecasting relies on historical data, therefore implicitly assuming that the future is accurately described by the past. However, given that demand patterns during COVID-19 and in the recovery phase are so different, "We've had to look at the forecast in a more holistic way... and relook at how we do the forecast, how we optimize passenger demand, and what sources of information are brought in" [18]. Before COVID-19, most RM decisions were made based exclusively on internal data (such as historic bookings). As airlines start leveraging external data sources, many new questions arise. What data sources should be used and how should they be integrated with historical data? Is there any value in using historic booking data during COVID-19? After COVID-19, should airlines return to using just historical data, or are there approaches that can be used to integrate traditional and new data sources?

Several characteristics about the booking curve, such as demand volumes and the pickup curves (or when bookings are occurring relative to the departure date), have changed with the pandemic [19]. Airlines and researchers have been exploring a variety of new data sources to better capture these effects. Lufthansa incorporated into its forecasts the number of COVID-19 cases and the percent of the population who have been vaccinated [11], and Southwest Airlines used the number of 
passengers passing through Transportation Security Administration (TSA) checkpoints [2]. However, by far, the most common source of new data that is being incorporated into demand forecasts is shopping data [19-21]. As Matson of Alaska Airlines states, "During the first and second wave in the United States, we saw a lot of correlation of bookings to searches of coronavirus dashboards. And the big breakout term for recovery was 'vaccine appointment.' We took those trends and incorporated them as one-week leading indicators for bookings" [17].

The benefit of using shopping data to improve forecasting accuracy in both preCOVID-19 and COVID-19 periods was discussed in several presentations. Lakshmanan [20] describes a model that Sabre developed to forecast market sizes. She starts with traditional data, namely historic monthly passenger and fare data, and prepares forecasts using traditional time-series-based methods, including a seasonal naïve model and autoregressive integrated moving average (ARIMA) model. She then incorporates machine learning (ML) methods, specifically the extreme gradient boost (XGBoost) algorithm that combines lagged data with various levels of lag to generate predictions. Based on pre-COVID-19 data, the XGBoost algorithm produces a forecast that has a mean absolute percentage error (MAPE) of 18.2, which is lower than the MAPE of 35.9 for the naïve method and 23.8 for the ARIMA method. Also using the XGBoost algorithm, Lakshmanan incorporates historic online shopping data for airline itineraries and finds that the model based solely on historical data has a MAPE of 17.7 compared to the model that uses both historical and shopping data that has a MAPE of 6.3; error measures were based on predicting market size for the 250 largest markets in North America [20]. This is one example of how online search data and ML techniques have been particularly valuable as a new data source for demand prediction during COVID-19 and will likely become a common data source for demand prediction after COVID-19.

In another example, Dietrich, Etabarialamdari, and Wilson [22] describe a demand forecasting model that Air Canada was using for its RM system before COVID-19 and then adapted during the pandemic. The main objective of their approach is to improve the accuracy of the forecasts of the number of remaining unconstrained bookings for an origin-destination (OD) at various days before departure and provide guidance to revenue management analysts as to where and why a forecast generated using traditional time series methods could be improved. The authors use ML methods, including a recurrent neural network, to generate a forecast that has two key inputs: (1) traditional time series data, and (2) other features. They compare the forecasts generated from the traditional approach and the ML approach to identify the forecasting data points that are most different, then identify specific characteristics that help explain these differences and recommend adjustments back into the RM system to the analyst. For example, if the ML forecast shows that the traditional forecast underestimates demand for Monday flights departing two to three weeks from departure, a recommendation can be generated for how the analyst can adjust the demand inputs to the RM system. For pre-COVID-19 data, this approach improved forecasting accuracy by more than 25 percent on average across 60 OD pairs at Air Canada. During COVID-19, their approach was modified so that the ML algorithm used not only historic data, but synthetically generated data that represented different demand scenarios to help test the sensitivity of results to different 
recovery levels. The use of synthetically generated data to generate bid prices under different demand scenarios was also discussed by Rauch [23].

An interesting part of the approach that Air Canada has taken is that the ML forecast was not designed to replace the existing RM algorithms, but rather to provide recommendations to analysts; thus, it is ultimately up to the analyst to decide whether or not to implement the recommendation (or implement a modification based on the recommendation). This philosophy was shared across multiple airlines, in part, because while "there's no shortage of data out there, the shortage is our ability to ingest and interpret the data and make it valuable for us" [24] and, in part, because when all of these data sources are considered "sometimes it contradicts itself and someone has to make up his mind on it" [11]. For example, Bradshaw of American Airlines describes how their demand forecasts for schedule planning applications have changed, as follows: "It may not necessarily be using one or two traditional data sources, but trying to incorporate little bits and complement what you may have. So, you start with traditional booking data out of MIDT ${ }^{5}$, but then you may get shopping data either from Google or from other places and see if the data are giving you the same directional result" [14].

The example of Dietrich, Etabarialamdari, and Wilson [22] is important in the context of how RM systems may evolve post-COVID-19. In Air Canada's case, the existing RM system, optimization algorithms, and data inputs are maintained, but the data inputs are adjusted by analysts based on analyses that are conducted external to the RM system. Conceptually, this approach could be adapted so that the supplemental analyses consider other external data sources (such as shopping data) when producing recommendations.

Given that the use of nontraditional data sources in RM systems is so new, it should not be surprising that airlines differ in their perspectives on how they will use these supplemental data sources moving forward. It is unclear at this time whether the airline industry will continue to rely on analyst intervention in the future and/or whether the data that are used for the ML methods will become reliable enough to use with minimal oversight. Multiple airlines emphasized that they would not want the RM system optimized for black swan events [10, 25], but there would be value in incorporating some of the new data sources into future RM systems. With respect to other data sources, Lucio Bustillo of Air Canada explains, "Once the bookings are back, the question becomes which of all the sources that we've explored are really something that in steady state brings something meaningful in terms of accuracy and improvement for the forecast...We've found the economic data, consumer sentiment, and search data to be very helpful for understanding the underlying travel intent, and we've seen correlations between a spike in searches and a wave of bookings a week or two later. So, there's value long-term on using those data" [18].

A key question being explored by the airline community that ties in closely to whether the traditional RM systems will be viable in the new normal after COVID19 is whether historical data provide useful information and can be used in forecasts.

\footnotetext{
5 Marketing Information Data Tapes (MIDT) data contain reservation data captured by travel agents from major global distribution systems.
} 
Fiig and Wittman [19] examine how historical data could be used for forecasting demand during COVID-19. They decompose the forecasting problem into two components: those that are resilient and those that are volatile. Resilient components characterize demand patterns that remain stable over the pandemic (e.g., in the USA, individuals still want to travel over Thanksgiving). Monthly seasonality patterns and day-of-week patterns have also remained stable over the pandemic. Volatile components, or those that have changed during the pandemic include market sizes, or the volume of bookings, and when these bookings are occurring, or the pickup curve. They use live shopping data to model these volatile components and automatically adjust the historic forecast. The approach of Fiig and Wittman is particularly relevant for airlines during the COVID-19 recovery period as it provides one approach for using historical data in a traditional RM system by addressing a shortcoming of current demand forecasters that assume demand volatility does not exist.

Looking beyond COVID-19, many airlines are seeking new approaches to adapt their RM systems to an environment in which many of the products offered are flexible and refundable. As a result of fundamental demand shifts that will remain after the pandemic, many airlines emphasized that they were viewing COVID-19 "as a catalyst for change" [26] and shifting development priorities. As discussed in [27], Air Canada, United, and Qatar all indicated a greater focus being placed on dynamic pricing and continuous pricing [26, 28, 29]. As Dennis Buitendijk of Qatar summed up, "The one good thing about a crisis is people now see why [dynamic or continuous pricing] is relevant and how it would complement a[n] RM system," [26] as quoted in [27]. Emirates provides an example of how have they are using shopping data to support dynamic availability and pricing decisions [30].

In summary, the "pandemic really shook up some of us and forced us to go out of the comfort zone when it comes to the data" that is used for forecasting demand [18]. Research is needed to help identify new data sources that can be used for forecasting airline demand, as well as which forecasting methods provide accurate solutions over time. It is likely that shopping data will become an integral part of forecasting airline demand and that researchers will leverage insights they have gained from using shopping data in other applications, such as generating personalized air ancillary offers in real time [31] or for dynamic availability and pricing [30] to help inform demand forecasting for RM and schedule planning applications. There is a research need to examine how dynamic and continuous pricing can complement existing RM systems in a post-COVID-19 environment.

\section{Schedule Planning in the COVID-19 Era}

During COVID-19, it has been challenging for airlines to build future schedules and decide how much capacity to put in different markets. Before COVID-19, many airlines planned their schedules six to nine months from departure, but as Bradshaw of American Airlines states, "That went out the window in 2020...we've had to get very flexible in how we schedule the airline to try to match up capacity with demand and make schedule changes as close in as 30 days, or even three weeks out from departure in some cases" [14]. 
Those airlines that had tools that allowed them to design their schedules from scratch and/or automatically cut a large number of flights from their schedules were in a better position to realign their schedules to match demand. For example, before COVID-19, Southwest Airlines had implemented a clean-sheet schedule optimization that allowed them to "start from scratch with optimization...and basically pick up our entire schedule and move it wherever we needed to go" [24]. Adam Decaire explains that Southwest Airlines used this tool in late March 2020, when demand was falling 90 percent or more year-over-year, to publish a new schedule for early May. The new schedule better matched supply with customer demand-but also better realigned crew with equipment and improved on-time performance. "Back in April [2020] our on-time performance was down to like 40 percent. And then all of a sudden at May, we're at 90 percent and above. That was a brand-new schedule completely written for the information we had at the time, not a schedule that was written 200 days ago" [2].

Ironically, Southwest Airlines had another unexpected event in 2019 that better prepared them for optimizing schedules during COVID-19, i.e., "the grounding of the MAX got us ready for the pandemic as we were the largest operator of the MAX aircraft and, at some point, we had to pull 60 aircraft out of our schedule and do that in the least disruptive way to our customers and employees" [2]. During this time, Southwest developed tools that helped them identify which airplanes to take out of the schedule and which flights to move, and put in processes to inform customers very quickly of the changes that had been made to their itineraries. All airlines faced a need to replicate these processes during COVID-19.

Airlines have taken different approaches to how they schedule flights during the pandemic, but one trend prevalent across many airlines is that they are initially publishing a schedule assuming that demand will come in strong, and then weeks and/ or days from departure, they make changes to the schedule to better match supply to demand. This may involve canceling some flights (and reaccommodating passengers on other flights), swapping equipment types (to provide more or less seats on a given flight leg), or adding extra sections (in situations where flights are sold out). Making all of these changes close to departure presents new challenges for airlines, particularly with respect to crew planning and operations.

Before explaining how these close-in schedule changes are driving new research needs in crew planning and airline operations, though, it is helpful to understand why airlines are using the approach of setting a future schedule under an assumption that demand will come in strong. Baharnemati, Bray, and Hurwitz [32] describe an analysis they conducted to understand how Southwest Airlines' capacity decisions influenced that airline's load factors and revenue projections compared to factors that were outside of their control, such as passenger demand levels and the capacity offered by competing airlines. They found that Southwest's own capacity decisions had a small impact on Southwest's load factors and revenue projections compared to assumptions related to passenger demand and their competitors' capacity decisions. As noted by Hurwitz, "In the end, [our analysis] really helped everyone understand it's not risky to go with our bigger capacity set. Relatively speaking, with everything that is out of our control versus that is in our control, we'd rather have those flights out there" [32]. 
In summary, COVID-19 is driving new research needs related to scheduling of flights. Given uncertainty and volatility in passenger demand, airlines are no longer able to publish a (relatively static) schedule months in advance of departure. Designing schedules that are robust to different demand scenarios and that maintain flexibility for downline processes, such as crew planning and operations recovery, are important areas for new research.

\section{Crew Planning in the COVID-19 Era}

The need for flexibility and close-in scheduling (and rescheduling) of flights is driving new research needs in crew planning. Crew planning involves several components, including manpower planning. Manpower planning involves determining how many crew an airline will need in the near-term and long-term and helps inform when the airline should start hiring and training crew for different fleets. PreCOVID-19, the majority of airlines did manpower planning in spreadsheets, but during COVID-19 many of these airlines have moved toward using software designed to optimize manpower planning. While this may seem surprising, as shown in Fig. 1, globally there are many more "small airlines" than "big airlines." Worldwide, more than 1,000 airlines are in operation and, among these, only 2 percent have fleet sizes of 200 aircraft or more, whereas 78 percent have fleet sizes of 25 or fewer aircraft. The largest 2 percent of airlines operate 22 percent of all aircraft, and the smallest 78 percent operate 31 percent of all aircraft [33]. Before COVID-19, many of these smaller airlines could manually handle manpower planning, but during COVID-19, automating manpower optimization became critical as airlines faced furlough and lay-off decisions and wanted to consider alternate demand recovery scenarios before making these decisions.

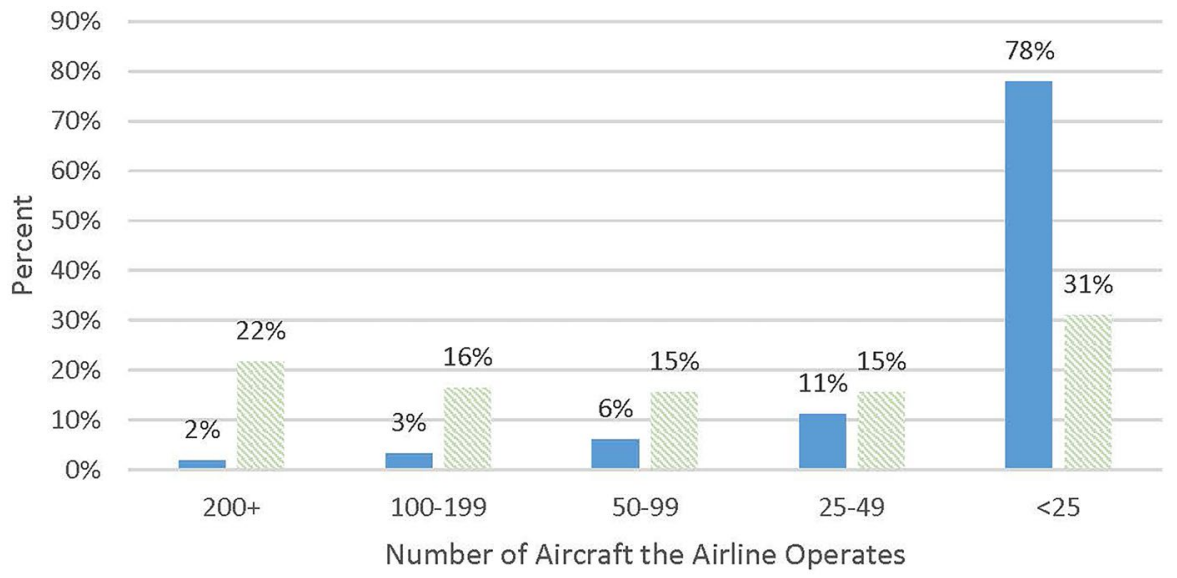

Airlines Total Aircraft

Fig. 1 Distribution of Worldwide Airline Fleet Sizes. Source: [33] 
The need for flexibility and close-in scheduling of flights is emphasizing new research needs in crew pairings, which is the process by which flight legs are combined together in a way that start and end in a crew base and are later combined into crew rosters. New research in crew pairings is being driven in large part because airlines "don't fly the same schedule every day of the week" [24] or from week to week. As Soumis [34] explains, the crew-pairing problem has evolved from where a similar flight schedule was offered every day and a problem size may have involved a few hundred flights, to a monthly problem in which flight schedules vary week to week and thousands of flights need to be considered. The monthly crew-pairing problem is particularly challenging, as it is very large and has no cyclical structure. Soumis and colleagues [34] have proposed using ML in combination with traditional OR models to find good solutions for the monthly crew-pairing problems. Soumis and colleagues use ML to predict good crew connections between inbound and outbound flights at an airport that have a high probability of being included in an optimal or near-optimal pairing solution. The ML model is a supervised multi-layer (1-5 layer) convolutional neural network with 100-1000 neurons per layer. While this model can be time-consuming to solve, it can be solved well in advance. They then create clusters that combine connections with the highest such probabilities to create small sequences of flights. These are fed into a math programming model to find full pairings with other connections that complement the existing good connections. They devise a pricing problem that further improves these by iteratively breaking some of the existing clusters and creating near-optimal pairings. While the ML model in itself is not good at solving for the optimal pairings and only provides upper bounds, it does predict good connections, which when fed into a math programming model, provide high benefits in reducing the size and solution time of the math programming model, and generates provably near-optimal solutions [34].

Other researchers, including Lingaya and Dashora [35], are also exploring new approaches to the robust crew-pairing optimization based on a monthly schedule. Currently, there is no convergence in the field as to how to define "robustness," and multiple metrics are considered. In their work, Lingaya and Dashora [35] examine robustness in crew pairings with respect to how often cabin crew followed the flight deck, regularity in the pairings produced, and block time variation.

COVID-19 has also impacted how airlines create rosters. Some airlines, including Indigo and Cathay Pacific have introduced new key performance indicators (KPIs) that limit the number of crew members that an individual comes in contact with [36, 37]. Ensuring that recency requirements are $\operatorname{met}^{6}$ has also been a challenge for many airlines. As Andy Spillane of British Airways explains, COVID-19 brought "an unprecedented amount of change...and we had to get a lot of staff off of payroll and onto furlough. You're trying to spread that out fairly, as well, and keep your crew recent, as all of a sudden, you've got far too many aircraft and far too many crew. So, you start getting recency issues that you've never experienced before" [1]. Creating

\footnotetext{
${ }^{6}$ Recency requirements refer to tasks or items that must have "recently" been completed by a crew member in order to maintain their ability to fly. For example, a pilot must execute a certain number of takeoffs and landings within the previous 90 days.
} 
rosters that take into account recency requirements and/or adding in additional simulation training that can fulfill recency requirements has become more common at airlines, including Cathay Pacific [38].

In addition, COVID-19 has impacted when crews receive their rosters. Many airlines have union contracts that dictate what percent of the roster may change and how far in advance the rosters need to be published. Conceptually, these rules help crew achieve a better work-life balance. For example, when crew know in advance which days they will be off next month, they can schedule medical appointments, purchase tickets to a sporting event, plan a birthday party, etc. COVID-19 has made it difficult for airlines to assign crew to specific flights a full month in advance of departure, as passengers are booking much closer to departure, borders are opening and closing, new travel restrictions that affect crew layovers may go into effect, etc. All of these factors result in major schedule changes that occur close to departure.

From the airlines' perspective, it would be ideal to assign crew to flights as close to flight departure as possible; however, from the crew's perspective, this becomes a quality-of-life issue that limits their ability to plan events on their days off. Instead of fixing the rosters a month in advance of departure, some airlines have adopted a "continuous publication" approach. Under this framework, certain details of the roster, e.g., the days a crew member will have off, are published or finalized three to four weeks from departure, but other details_-like the specific flights-are published one to two weeks from departure [39]. This enables airlines to maintain flexibility in scheduling flights while providing crew with information regarding their days off. Offering information on days off further in advance of departure has other benefits, as well, e.g., it reduces the number of crew sick days.

This is one example of how airlines have been able to balance the need for flexibility with the desire of crew to know their assignments a month or more before departure. Other areas of research that are emerging during COVID-19 are: (1) Incorporating other crew preferences into the rostering process, and (2) ensuring that crew rosters are fairly assigned across individuals. For example, Scherp, Beulen, and Santos [40] propose a dynamic crew-rostering framework in which crews can request to receive a specific pairing assignment before the rosters are published. If the request is accepted, the airline has to include that pairing in the roster for the next month. While pre-assigning these requests before the entire roster is built can result in more expensive solutions, the ultimate goal is to identify those crew pairings that are most likely to be in the final roster and assign those in advance without compromising the full roster by leaving gaps in the schedule or requiring more crew to fly.

The work of Scherp, Beulen, and Santos is important because the feedback for these requests is given to crew before the actual rostering process starts. The more traditional way of doing this is to have the crew enter bids in the weeks before rostering starts, have the optimizers take these bids into account when creating the optimal rosters, and inform the crew of whether their bids were successful at the moment of publication. At this point, it is too late for the crew to make any alterations to their bids. In the approach proposed by Scherp, Buelen, and Santos, crew place their requests and are informed either immediately or the day after placing their request whether it was accepted, thereby providing crew with the ability to 
place an alternate request if their original one was rejected. Historically, those airlines such as KLM that have provided these types of requests have used simple rules that either restrict the types of request crews can make or result in solutions that are inefficient. Scherp, Buelen, and Santos [40] propose an approach that overcomes these two limitations through better prediction of these inefficiencies and basing the decision to grant request on more complex logic. This is important in the context of COVID-19 as it provides a way for crew to gain control over their work-life balance, while simultaneously introducing flexibility into the rosters and supporting a continuous publication approach.

COVID-19 is, moreover, spurring new research in fatigue risk management [41, 42]. The opening and closing of borders, combined with quarantine rules for airline crew who deboard the aircraft, have forced some airlines to perform humanitarian flights that are pushing the boundaries on how long crew are flying and on duty. For example, Azul Airlines made humanitarian flights into China. A flight from Amsterdam to China and back to Amsterdam without a layover took about 30 hours; clearly, both the duty times and flight times for this trip exceeded the approved times. In advance, Azul Airlines worked with their fatigue management risk team to put together an eight-pilot rotation. They also worked with researchers who were using wearable sleep measurements (collected through devices such as a Garmin, Fitbit, Apple Watch, etc.) to improve fatigue risk management predictions [42]. The researchers captured information about when the pilots were actually sleeping and then compared pilots' actual sleep patterns with the planned or recommended sleep schedule and produced a customized report for every crew member. Azul Airlines found that "pilots loved these reports, as they were anxious to do a flight and understand how they reacted during the flight, and what they could do better to rest and be more attentive during the flight" [41].

This is one example of ongoing research by Hursh [42] that seeks to use feedback to pilots to create personalized fatigue analysis that ultimately can be used to develop better recommendations for when pilots should sleep based on an improved understanding of how flight schedules (under regular conditions) interact with pilots' sleep patterns. As airlines enter into the recovery mode from COVID-19, the speed in which the number of flights is coming back into the schedules is faster than the speed in which many airlines can bring back crew from furlough or rehire new crew. This creates a risk that some airlines may be inadvertently building too many flying hours into the monthly rosters. The value of incorporating bio-mathematical models into the rostering optimizers is that airlines can build additional flying hours into the monthly rosters while still ensuring that crew do not lose alertness due to fatigue, and thus ensuring safe operations.

In summary, because bookings during COVID-19 have fluctuated and are occurring close to departure, airlines have been making multiple changes to their flight schedules. In addition, many airlines have moved to operating schedules that differ day to day and week to week. Both of these factors have increased the size of the crew pairing and rostering problems and made them more difficult to solve. Further, instead of finding the optimal solution for a deterministic schedule, many researchers are beginning to explore how to design crew pairings and rosters that are robust to different demand and flight-schedule scenarios. Ensuring equity in duty 
assignments across crew members and using wearable technologies to help develop better fatigue risk management strategies are also emerging areas of research.

\section{Operations Recovery in the COVID-19 Era}

With all of the changes in schedules that are occurring prior to departure, it should be unsurprising that the role of airline operations and disruption management are also changing. At a high level, airline operations involve executing all of the different "schedules" that are involved in running an airline and reacting to the inevitable problems that arise from unexpected events that affect one or more of these schedules. These schedules include, but are not limited to, the flight schedules, aircraft assignment schedules, crew schedules for pilots and flight attendants, heavy maintenance and line maintenance schedules, and aircraft turn schedules.

COVID-19 brought several changes to airline operations. On one hand, because the number of operated flights decreased during COVID-19, there was more slack in the schedules, which helped alleviate recovery of operations from weather-based events. However, because of ever-evolving standards regarding sanitary measures for aircraft and social-distancing measures for passengers, the turnaround process has become more complex and dynamic [43]. Many questions remain unresolved. For example, do we need additional cleaning of the aircraft and, if so, how long does that take? Should the boarding process be lengthened to allow for better social distancing?

As Mike Irrgang, chair of the AGIFORS Operations Planning Study Group notes, these and other questions have led to, "For the first time in 30 years, how we plan for aircraft turn times" [43]. This was demanded in large part by new cleaning procedures that were added between flights. Teixeira, Higa, Jakabi, and Halawi [44] use a simulation framework to model the impact of cleaning and other COVID-19-related procedures on aircraft ground times for the Congonhas Airport in Brazil. For ground times of less than 60 minutes, they find that ground time increased on average 8.1 minutes. Based on an empirical analysis, Rana and Gandotra [45] also find that ground times at United Airlines increased on average by 8 minutes. This resulted in the mean time spent by each flight in the airport system increasing by 7.7 minutes and the maximum time spent by a flight in the airport increasing by 5.7 minutes. While airlines are flying a reduced schedule, the impact of these increased ground times is minimal, but as airlines return to their pre-COVID-19 flight levels, it will be important to find ways to decrease these turn times in order to maintain higher aircraft utilizations.

In some areas of the world, government support provided opportunities for airlines to complete maintenance tasks ahead of schedule. As Garrett [46] of United Airlines explains, payroll support provided by the CARES $\mathrm{Act}^{7}$ in the USA provided excess maintenance labor in addition to what was necessary to operate a reduced schedule. Garrett and her colleagues designed an optimization model to determine

\footnotetext{
${ }_{7}$ Coronavirus Aid, Relief, and Economic Security Act
} 
which maintenance tasks not to do on aircraft because they were going to have to do them again when the aircraft returned to service, and which maintenance tasks to complete early even though they were not due because they would be due at critical times in the future when United was planning to ramp up service.

Another change that is occurring within airline operations is that planning for recovery is shifting to reactive operations recovery to mitigate the impact of events without advance notice (such as weather on the day of operations) to more proactive operations recovery, or planning for events with advance notice (such as dynamic schedule changes that occur one or more days in advance of departure). This presents a shift in how the airline industry needs to think about positioning aircraft and reserve crew. Before COVID-19, the focus was on recovering from flight cancellations that happened in real time and ensuring that crews returned to their bases in a legal fashion and aircraft back on schedule. During COVID-19, the recovery problem has changed to one of repositioning assets in advance of departure, e.g., moving aircraft and/or reserve crew to different stations to cover changes in the schedule and/or proactively canceling flights and removing them from the schedule while ensuring the aircraft return to bases in time for required maintenance checks. COVID-19 has caused changes in the interaction between the schedule planning and the operations recovery in that it has reduced the lead time to build a schedule, requiring airlines to be nimble and flexible in designing schedules for (mostly) leisure markets. Correspondingly, the scope of operations recovery has widened, to almost two months prior to the day-of-operations, while the schedule planning process is being curtailed. In some ways, disruption management is thus becoming "easier" in the sense that there is more lead time to plan for these disruptions, but it will require new ways of thinking about recovery. Prior experiences in proactively canceling and repositioning crew and flights before a major weather event, such as a snowstorm or major hurricane, are helping inform disruption planning during the COVID-19 recovery [47]. Airlines anticipate that the techniques of continuously monitoring the network to forecast disruptions, maintaining high situational awareness and dynamic scheduling to match capacity to demand, are all techniques that will help airlines during post-COVID operations.

In summary, COVID-19 is inducing new innovations and research needs within operations. The role of disruption management has shifted from planning for unexpected events on the day of departure to planning for events known several days in advance of departure. After the pandemic, airlines will likely be able to return to publishing their schedules a month in advance of departure with fewer changes; however, the use of scenario-planning tools will likely become more common as airlines plan their various schedules to handle different demand scenarios.

\section{A Need for Integrated Solutions that are Resilient and Robust}

One of the more positive outcomes that has been driven by COVID-19 is that different groups within an airline are working together to solve OR problems, resulting in more integrated solutions. These collaborations are seen throughout many areas that have been traditionally siloed. 
For example, the revenue management and scheduling departments are closely working together to align supply and demand. "There is a lot more back-andforth between revenue management and scheduling, particularly when it comes to really trying to identify where there are opportunities for demand" [28] and to support last-minute flight cancellations [48]. As airlines ramp back up their operations, the "fundamental assumption of RM that capacity is fixed does not hold true in times when large parts of an airline's fleet are on the ground and could be re-activated in case of undercapacity. This results in a need for more integrated solutions between RM and network planning" [49]. Designing the various schedules involved in operations planning in ways that incorporate new objectives or multiple objectives is becoming more important during COVID-19. For example, with respect to new objective functions, instead of having RM systems maximize revenue, many are considering cash flow (potentially prioritizing early earnings) and profitability (as different cost structures lead to different RM strategies) [49]. Numerous examples emerged of how airlines are incorporating multiple objectives into OR approaches. In the context of schedule planning, Matt Muehleisen of Southwest Airlines acknowledges that "trying to understand the profitability of the airlines as it relates to operating that schedule - so trying to bring in the crew factor and the operational factors and recovery factors into one modee - is the holy grail of network planning, and I don't think we're there yet" [24]. With respect to crew planning, Peter Reynolds of Emirates notes that "we have a very good roster optimizer that builds excellent rosters. But then we put them into our [operations] system and we tear them apart and try and put them back in some reasonable fashion to still get the best out of our crew...But what we really are crying out for is a way to quickly re-optimize our rosters so that all the work that goes into planning the perfect roster doesn't go to waste and you end up with wasted resources everywhere" [50]. Multiple presentations at AGIFORS discussed approaches for breaking down barriers between the pairing-rostering and crew-control silos, including the work by Rosterize that solves this integrated solution for small airlines [51].

Several of the presentations at the AGIFORS conferences explored ways to integrate decisions made within various silos of the schedule-planning process, such as schedule planning, fleet assignment, aircraft routing and scheduling, and air traffic flow management. For example, Yan, Vaze, and Barnhart [52] propose an integrated schedule design and fleet assignment model that considers passenger preferences (e.g., flight departure time preferences and price sensitivity). Their model helps determine how many flights to operate in a market, when to operate these flights, and which fleet type to assign to these flights. Çiftçi and Ozkir [53] examine the schedule-generation process using a dataset from a Turkish carrier to create efficient bank structures by jointly considering the schedule design and fleet assignment models. Some airlines, particularly those in Europe, studied their schedule planning and fleeting decisions in the context of air traffic flow management initiatives that require schedules to be flattened to avoid congestion and delays [54]. The authors find that significant reductions in congestion can be achieved through small changes in frequencies and increases in aircraft sizes. This leads to decreases in passenger travel times, but it does not have a large influence on emissions [54]. 
In summary, COVID-19 is spurring new research into how to incorporate resiliency and robustness into OR problems. COVID-19 has also broken down traditional silos, as analysts from scheduling, revenue management, sales, operations, and other areas worked together, which presents new opportunities for pursuing more integrated solutions across the functional areas.

\title{
8 Summary of Research Opportunities
}

The airline industry is without doubt being reshaped by the COVID-19 pandemic. Fundamental changes in passenger demand and booking patterns are driving the need for new operations research (OR) solutions and potentially will lead to fundamental changes in forecasting, revenue management, and capacity allocation decisions. Interest in using dynamic pricing and continuous pricing is surging. New research is emerging across multiple areas. Researchers are using new data sources and scenario-based methodologies to forecast demand, are exploring techniques such as clean-slate scheduling to quickly realign supply with demand, and solving very large-scale crew pairing and rostering problems that consider monthly flight schedules. The role of operations recovery planning has also expanded as many airlines make changes to their schedules a mere two or three days in advance of departure to better align supply with demand. A common theme throughout many of these approaches is the need for solutions that are robust to different demand scenarios and/or that offer integrated solutions across multiple functional areas.

\begin{abstract}
Acknowledgements The authors thank all the speakers who participated in the conference, as well as Dennis Buitendijk, Judith Semar, Cumhur Gelogullari, Marcel Sol, Philipp Reske, Daniel Stecher, and members of the AGIFORS Advisory Board who helped recruit speakers and/or moderate panel sessions. The authors are also grateful to Debbie Fleming of Administrative Plus Support Services for transcribing the panel and keynote sessions, Sharon Dunn who copyedited the manuscript prior to submission, and Freyja Brandel-Tanis who assisted with references. The authors also wish to thank Benedikt Zimmerman of Swiss International Air Lines, Richard Cleaz-Savoyen of Air Canada, Marcel Sol and Dennis Buitendijk of Qatar Airways, and Mike Irrgang and Rod Tjoelker of Boeing for their detailed comments on an earlier draft of this paper.
\end{abstract}

Author Contributions All authors contributed equally to this article.

\section{Funding None.}

Data Availability The majority of recorded presentations from the conference are available online at the following locations: Revenue management: https://www.youtube.com/playlist?list=PLCZDWSD0js_ tLJq2b9LwKqm8By24vvjxu. Strategic planning and scheduling: https://www.youtube.com/watch?v= oOP7LpfaNFk?list=PLWgDdcExmf7N1kwHRrNSZYg9TWlzWaJ1Q. Crew management: https://www. youtube.com/playlist?list=PL1S9gXa91S6OgnktHNyAdzTa-qCfKypqr. Airline operations and aircraft maintenance operations: https://www.youtube.com/playlist?list=PLE8k3MOnJTMZu0Ui89kAexb2 Vv4Oeta6A. In addition, presentations are available through the AGIFORS publication database at http:// www.agifors.org and are accessible to AGIFORS members. Membership in AGIFORS is free.

Code Availability Not applicable. 


\section{Declarations}

Conflicts of Interest/Competing Interests Dr. Garrow and Dr. Marla serve on the Board of Directors for AGIFORS and Dr. Lurkin is Co-Chair of the AGIFORS Revenue Management Study Group.

\section{References}

1. Spillane A (2021) Global operations change manager at british airways. Panelist inGlobal Airline Industry Trends from Across the World in 2020: Which Ones Are Here to Stay? AGIFORS Crew Management Study Group Meeting, June 15, virtual event

2. Decaire A (2021) Vice president of network planning at southwest airlines. Keynote speaker at AGIFORS Scheduling and Strategic Planning Study Group Meeting, May 17, virtual event

3. Jönsson P (2021) Jeppensen crew management. Sponsor presentation at the AGIFORS Crew Management Study Group Meeting Meeting, June 10, virtual event

4. Delta Air Lines (2021) Delta Air Lines announces June quarter 2021 financial results. Delta News Hub. https://news.delta.com/delta-air-lines-announces-june-quarter-2021-financial-results. Accessed 4 Aug 2021

5. Anonymous (2021, August 4) Spirit AeroSystems reports second quarter 2021 results. Businesswire. https://www.businesswire.com/news/home/20210804005415/en/Spirit-AeroSystems-ReportsSecond-Quarter-2021-Results. Accessed 12 Sept 2021

6. Rucinski T, Ajmera A (2021) American and Southwest lifted by 'messy' travel rebound, federal aid. Reuters. https://www.reuters.com/business/aerospace-defense/american-southwest-post-profitsjune-even-without-federal-aid-2021-07-22. Accessed 4 August 2021

7. Sebastian D (2021) JetBlue turns 2Q profit. MarketWatch. https://www.marketwatch.com/story/jetblueturns-2q-profit-271627385869. Accessed 4 Aug 2021

8. Wolfsteller P (2021) Allegiant posts Q2 profit, expresses optimism for the rest of 2021. FlightGlobal. https://www.flightglobal.com/strategy/allegiant-posts-q2-profit-expresses-optimism-for-therest-of-2021/144819.article. Accessed 4 Aug 2021

9. Airlines for America (2021) Impact of COVID-19: Data updates. Airlines for America.https://www. airlines.org/dataset/impact-of-covid19-data-updates/. Accessed 7 Sept 2021

10. Juhasz B (2021) Senior Manager Operations Research and Insights at Finnair. Panelist in Airline Revenue Management and COVID-19: Road to Recovery. AGIFORS Revenue Management Study Group Meeting, May 5, virtual event

11. Bharpalani M (2021) Head of Data and Analytics at Lufthansa Group. Panelist in How to Fight the Pandemic and Navigate to Recovery. AGIFORS Scheduling and Strategic Planning Study Group Meeting, May 18, virtual event

12. Correia R (2021) Senior Director of Itinerary Planning at LATAM Airlines. Panelist in How to Fight the Pandemic and Navigate to Recovery. AGIFORS Scheduling and Strategic Planning Study Group Meeting, May 18, virtual event

13. Pekesen E (2021) Senior Vice President of Sales and Network Planning at Pegasus Airlines. Panelist in How to Fight the Pandemic and Navigate to Recovery. AGIFORS Scheduling and Strategic Planning Study Group Meeting, May 18, virtual event

14. Bradshaw T (2021) Director of Models and Systems, Network Planning at American Airlines. Panelist in How to Fight the Pandemic and Navigate to Recovery. AGIFORS Scheduling and Strategic Planning Study Group Meeting, May 18, virtual event

15. Garrow LA, Lurkin V (2021) How COVID-19 is impacting and reshaping the airline industry. J Revenue Pricing Manag 20(1): 3-9. https://doi.org/10.1057/s41272-020-00271-1

16. Pallini $\mathrm{T}$ (2020) JetBlue just announced a huge nationwide expansion that sees 24 new routes and 4 new cities in 2021 with more flying to Latin America - here's the full list. Business Insider. https:// www.businessinsider.com/jetblue-adds-24-new-routes-4-new-cities-new-year-2020-12. Accessed September 7, 2021

17. Matson A (2021) Senior Manager Data Science at Alaska Airlines. Panelist in Airline Revenue Management and COVID-19: Road to Recovery. AGIFORS Revenue Management Study Group Meeting, May 5, virtual event 
18. Bustillo L (2021) Director Revenue Management - Science and Technology Strategy at Air Canada. Panelist in Airline Revenue Management and COVID-19: Road to Recovery. AGIFORS Revenue Management Study Group Meeting, May 5, virtual event

19. Fiig T, Wittman M (2021) Revenue management forecasting in times of change: Lessons learned from a year into the pandemic. Presentation at the AGIFORS Revenue Management Study Group Meeting, May 5, virtual event

20. Lakshmanan A (2021) Market demand forecasting. Presentation at the AGIFORS Strategic Scheduling and Planning Study Group Meeting, May 17, virtual event

21. Winegar R, Wu M (2021) What can airlines learn from retailers about demand forecasting. Presentation at the AGIFORS Revenue Management Study Group Meeting, May 6, virtual event

22. Dietrich C, Etabarialamdari N, Wilson P (2021) Crystal Ball 2.0 for passenger demand: Leveraging AI to power the calibration workflow. Presentation at the AGIFORS Revenue Management Study Group Meeting, May 4, virtual event

23. Rauch J (2021) Scenario-driven RM using simulation-based reinforcement learning. Presentation at the AGIFORS Revenue Management Study Group Meeting, May 4, virtual event

24. Muehleisen M (2021) Senior Director of Network Initiatives, Network Planning at Southwest Airlines. Panelist in How to Fight the Pandemic and Navigate to Recovery. AGIFORS Scheduling and Strategic Planning Study Group Meeting, May 18, virtual event

25. Mathews J (2021) Director Continuous Improvement and Enterprise Optimization at United Airlines. Panelist in Airline Revenue Management and COVID-19: Road to Recovery. AGIFORS Revenue Management Study Group Meeting, May 5, virtual event

26. Buitendijk D (2020) Manager operations research at Qatar airways. Panelist in COVID 19: A Chance to Reset Revenue Management Practices? AGIFORS 60th Annual Symposium, October 20, virtual event

27. Garrow LA (2020) The first 100 days: How airlines responded to the Covid 19 crisis. Avionics International, Aug/Sep. http://interactive.aviationtoday.com/avionicsmagazine/august-september-2020/thefirst-100-days-how-airlines-responded-to-the-covid-19-crisis

28. Cleaz-Savoyen R (2020) Director, revenue optimization at Air Canada. Panelist in COVID 19: A Chance to Reset Revenue Management Practices? AGIFORS 60th Annual Symposium, October 20, virtual event

29. Ruhlin E (2020) Managing Director Revenue Decision Support at United Airlines. Panelist in COVID 19: A Chance to Reset Revenue Management Practices? AGIFORS 60th Annual Symposium, October 20, virtual event

30. Dener E, Komirishetty G, Ratliff R (2021) Practical applications of fare search shopping data. Presentation at the AGIFORS Revenue Management Study Group Meeting, May 6, virtual event

31. Ratliff R (2021) An approach to airline offer management: Dynamic bundling and pricing. Presentation at the AGIFORS Revenue Management Study Group Meeting, May 5, virtual event

32. Baharnemati R, Bray K, Hurwitz L (2021) Case study - Taking network forecasting accuracy to the next level during the COVID crisis. Presentation at the AGIFORS Strategic Scheduling and Planning Study Group Meeting, May 19, virtual event

33. Irrgang M (2019) Distribution of worldwide tails as of 2019. Data compiled by Boeing

34. Soumis F (2021) Machine learning and optimization to solve very large crew pairing problems. Presentation at the AGIFORS Crew Management Study Group Meeting, June 7, virtual event

35. Lingaya N, Dashora Y (2021) Towards robust crew pairing optimizer. Presentation at the AGIFORS Crew Management Study Group Meeting, June 10, virtual event

36. Forsman V (2021) Crew quarantine: A scheduling crisis for Cathay Pacific cargo. Presentation at the AGIFORS Crew Management Study Group Meeting, June 8, virtual event

37. Kumari A (2021) Director Crew Planning at Indigo Airlines. Panelist in Global Airline Industry Trends from Across the World in 2020: Which Ones Are Here to Stay? AGIFORS Crew Management Study Group Meeting, June 15, virtual event

38. Ozbek G (2021) Product Expert at Cathay Pacific. Panelist in Global Airline Industry Trends from Across the World in 2020: Which Ones Are Here to Stay? AGIFORS Crew Management Study Group Meeting, June 15, virtual event

39. Sol M (2021) Co-chair of the AGIFORS Crew Management Study Group. Interview with Laurie Garrow on July 28, 2021

40. Scherp L, Buelen M, Santos B (2021) Dynamic evaluation of airline pilots flight requests. Presentation at the AGIFORS Crew Management Study Group Meeting, June 7, virtual event 
41. Garcia C (2021) COVID-19 humanitarian operations at Azul. Presentation at AGIFORS Crew Management Study Group Meeting, June 17, virtual event

42. Hursh S (2021) Wearable sleep measurements to improve fatigue risk management. Presentation at AGIFORS Crew Management Study Group Meeting, June 7, virtual event

43. Irrgang M (2021) Chair of the AGIFORS Airline Operations Study Group. Interview with Laurie Garrow on July 26, 2021

44. Teixeira F, Higa F, Jakabi R, Halawi L (2021) COVID-19 impact on aircraft ground time at Congonhas Airport (CGH). Presentation at the AGIFORS Airline Operations Study Group Meeting, July 13, virtual event

45. Rana N, Gandotra M (2021) Turn operations in the post-pandemic era. Presentation at the AGIFORS Airline Operations Study Group Meeting, July 14, virtual event

46. Garrett L (2021) Leveraging analytics to create a dynamic technical operations strategy for storing aircraft and returning them to service. Presentation at the AGIFORS Aircraft Maintenance Operations Special Session, July 15, virtual event

47. Stephens E (2021) Dynamic scheduling lessons learned during COVID era in the U.S. Presentation at the AGIFORS Airline Operations Study Group Meeting, June 16, virtual event

48. Westerhof A (2020) Manager Revenue Management Systems at Air France-KLM. Panelist in COVID 19: A Chance to Reset Revenue Management Practices? AGIFORS 60th Annual Symposium, October 20, virtual event

49. Zimmerman B (2021) Member, Board of Directors of AGIFORS. Email communication with Laurie Garrow on September 14, 2021

50. Reynolds P (2021) Manager Network Ops Systems and Logistics at Emirates. Panelist in Global Airline Industry Trends from Across the World in 2020: Which Ones Are Here to Stay? AGIFORS Crew Management Study Group Meeting, June 15, virtual event

51. Andreyev M (2021) Rosterize. Sponsor presentation at the AGIFORS Crew Management Study Group Meeting, June 10, virtual event

52. Yan C, Vaze V, Barnhart C (2021) Choice-based airline schedule design and fleet assignment. Presentation at the AGIFORS Strategic Scheduling and Planning Study Group Meeting, May 17, virtual event

53. Çiftçi E, Ozkir V (2021) Fleet assignment with bank structure integration in airline schedule problem. Presentation at the AGIFORS Strategic Scheduling and Planning Study Group Meeting, May 19, virtual event

54. Presto F, Gollnick V, Lütjens K (2021) Frequency regulation: Approaches and potential. Presentation at the AGIFORS Strategic Scheduling and Planning Study Group Meeting, May 18, virtual event

Publisher's Note Springer Nature remains neutral with regard to jurisdictional claims in published maps and institutional affiliations.

\section{Authors and Affiliations}

\section{Laurie A. Garrow ${ }^{1}$ (D) Virginie Lurkin ${ }^{2} \cdot$ Lavanya Marla $^{3}$}

Virginie Lurkin

virginie.lurkin@unil.ch

Lavanya Marla

lavanyam@illinois.edu

1 School of Civil and Environmental Engineering, Georgia Institute of Technology, Atlanta, GA, USA

2 HEC Faculty of Business and Economics, University of Lausanne, Lausanne, Switzerland

3 Industrial and Enterprise Systems Engineering, University of Illinois at Urbana-Champaign, Urbana, IL, USA 